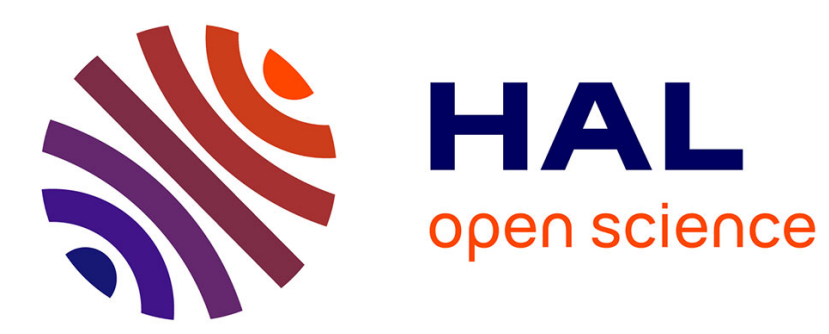

\title{
On the convergence of the proximal algorithm for nonsmooth functions involving analytic features
}

\author{
Hedy Attouch, Jerome Bolte
}

\section{To cite this version:}

Hedy Attouch, Jerome Bolte. On the convergence of the proximal algorithm for nonsmooth functions involving analytic features. Mathematical Programming, 2007, 116 (1), pp.5-16. 10.1007/s10107-0070133-5 . hal-00803898

\section{HAL Id: hal-00803898 \\ https://hal.science/hal-00803898}

Submitted on 23 Mar 2013

HAL is a multi-disciplinary open access archive for the deposit and dissemination of scientific research documents, whether they are published or not. The documents may come from teaching and research institutions in France or abroad, or from public or private research centers.
L'archive ouverte pluridisciplinaire HAL, est destinée au dépôt et à la diffusion de documents scientifiques de niveau recherche, publiés ou non, émanant des établissements d'enseignement et de recherche français ou étrangers, des laboratoires publics ou privés. 


\title{
On the convergence of the proximal algorithm for nonsmooth functions involving analytic features.
}

\author{
Hedy ATTOUCH ${ }^{1}$ Jérôme BOLTE ${ }^{2}$,
}

\begin{abstract}
We study the convergence of the proximal algorithm applied to nonsmooth functions that satisfy the Łojasiewicz inequality around their generalized critical points. Typical examples of functions complying with these conditions are continuous semialgebraic or subanalytic functions. Following Łojasiewicz's original idea, we prove that any bounded sequence generated by the proximal algorithm converges to some generalized critical point. We also obtain convergence rate results which are related to the flatness of the function by means of Łojasiewicz exponents. Apart from the sharp and elliptic cases which yield finite-time or geometric convergence, the decay estimates that are derived are of the type $O\left(k^{-s}\right)$, where $s \in(0,+\infty)$ depends on the flatness of the function.
\end{abstract}

Key words Proximal algorithm, Łojasiewicz inequality, real-analytic functions, subanalytic functions, convergence rate.

\section{Introduction}

The proximal algorithm has been first introduced by Martinet (1970) [21] and Rockafellar (1976) [23] as an approximation-regularization method in convex optimization and in the study of variational inequalities associated to maximal monotone operators. In the last decades, it has been successfully applied to a wide variety of situations, but still in the realm of convex optimization and monotone operators. Recent progress in the modelling of decision processes in economics and decision sciences (procedural rationality) provide strong motivation to develop the proximal algorithm in a nonconvex and possibly nonsmooth setting. Our main concern in the present paper is to develop this algorithm in such a general setting, namely by considering real-analytic functions, and more generally subanalytic lower semicontinuous functions. First, let us explain briefly the need to go beyond the classical convex setting and then, why analyticity features come naturally in the picture.

In [4] and [6], Attouch and Soubeyran developed a model for "real life" decision making which is an incremental decision process "A worthwhile to move approach of satisficing with not too much sacrificing". In this discrete dynamical model involving both exploration and exploitation aspects, the agent moves from a performance $x^{k}$ to $x^{k+1}$ when the estimated marginal gain $u\left(x^{k+1}\right)-u\left(x^{k}\right)$ is greater than, or equal to, the cost of moving $c\left(x^{k}, x^{k+1}\right)$. In this context, optimization features of the decision process are naturally modelled by the proximal algorithm (described below with the maximization version),

$$
x^{k+1} \in \operatorname{argmax}\left\{u(x)-c\left(x^{k}, x\right): x \in X\right\} .
$$

Classical proximal algorithms correspond to quadratic costs, i.e., $c(x, y)=|x-y|^{2}$ which expresses that small changes are costless. Because of the cost to change, this process becomes of local nature, which makes it more realistic than the classical global optimization modelling in economics and decision sciences.

The function $u$ measures the quality of the decision or performance $x \in X$, it is the utility function in economics, the valence in cognitive sciences. The opposite function $f=-u$, (which is now to minimize), measures how far is the current performance from a given long term goal. Indeed, the concavity of

\footnotetext{
${ }^{1}$ I3M UMR CNRS 5149, Université Montpellier II, Place Eugène Bataillon, 34095 Montpellier, France . email: attouch@math.univ-montp2.fr

${ }^{2}$ Equipe Combinatoire et Optimisation (UMR 7090), Case 189,

Université Pierre et Marie Curie, 4 Place Jussieu, 75252 Paris Cedex 05, France.

email: bolte@math.jussieu.fr
} 
$u$ (convexity of $f$ ) is a too restrictive assumption in order to cover many interesting applications: for example, in economics the utility function $\mathrm{u}$ is usually assumed to be quasiconcave. The convergence of the proximal algorithm for quasiconvex functions has been considered only recently, see Goudou-Munier [13], Attouch-Teboulle [5].

The proximal algorithm can be viewed as an implicit discretization of the continuous steepest descent method (also called continuous gradient method). This important fact has been soon recognized by many authors. It is at the origin of various developments which have been enriching the original algorithm and make it a powerful tool. A striking example is the link between interior point methods, proximal methods associated to Bregman functions, the Riemannian steepest descent and the Lotka-Volterra dynamical systems, see [5] and the references contained in it. An other example is the introduction of second order proximal methods, see [2], which are obtained as discrete versions of the heavy ball with friction dynamical system. This last system is an inertial version (second order dynamical system with respect to time) of the steepest descent, see [3]. Indeed, our special interest for the proximal method for functions involving analytic features comes from the recent developments concerning the convergence of the steepest descent method by Simon [25], Haraux [14] and Bolte-Daniilidis-Lewis [9]. In this last paper, the authors consider the case of subanalytic lower semicontinuous functions. This class of functions is very interesting because it covers many relevant problems in optimization and decision sciences (recall that, by the StoneWeierstrass theorem, polynomials of several variables and hence analytic functions are dense in the space of continuous functions for the topology of the uniform convergence on bounded sets). A key tool in the mathematical analysis of such continous or discrete dynamical systems is the Lojasiewicz inequality. It has been first stated by Łojasiewicz in the case of real-analytic functions [17] and, more recently, extended to nonsmooth functions [9].

Our main result (Theorem 4) relies precisely on a judicious use of the Eojasiewicz inequality and proves the convergence of the proximal algorithm to a critical point of the function to which it is applied ( $f$ or $u$ ). Based on Łojasiewicz's original idea [17] this type of results has already been applied successfully to explicit gradient method for analytic functions [1]. Our main result is completed by studying the rate of convergence of the algorithm (Theorem 5). This rate depends on the value of the so called Łojasiewicz exponents which can be thought as local measures of the flatness of functions around their generalized critical points.

\section{The proximal algorithm}

\subsection{Preliminaries}

The Euclidean scalar product of $\mathbb{R}^{n}$ and its corresponding norm are respectively denoted by $\langle\cdot, \cdot\rangle$ and $|\cdot|$.

Let us recall a few definitions concerning subdifferential calculus.

Definition 1 ([24]) Let $f: \mathbb{R}^{n} \rightarrow \mathbb{R} \cup\{+\infty\}$ be a proper lower semicontinuous function.

(i) The domain of $f$, written $\operatorname{dom} f$, is the subset of $\mathbb{R}^{n}$ on which $f$ is finite-valued.

(ii) For each $x \in \operatorname{dom} f$, the Fréchet subdifferential of $f$ at $x$, written $\hat{\partial} f(x)$, is the set of vectors $x^{*} \in \mathbb{R}^{n}$ which satisfy

$$
\liminf _{\substack{y \neq x \\ y \rightarrow x}} \frac{1}{|x-y|}\left[f(y)-f(x)-\left\langle x^{*}, y-x\right\rangle\right] \geq 0 .
$$

If $x \notin \operatorname{dom} f$, then $\hat{\partial} f(x)=\emptyset$.

(iii) The limiting-subdifferential $([22])$ of $f$ at $x \in \mathbb{R}^{n}$, written $\partial f$, is defined as follows

$$
\partial f(x):=\left\{x^{*} \in \mathbb{R}^{n}: \exists x_{n} \rightarrow x, f\left(x_{n}\right) \rightarrow f(x), x_{n}^{*} \in \hat{\partial} f\left(x_{n}\right) \rightarrow x^{*}\right\} .
$$


Remark 1 The above definition implies that $\hat{\partial} f(x) \subset \partial f(x)$, where the first set is convex while the second one is closed.

Remark 2 Clearly a necessary condition for $x \in \mathbb{R}^{n}$ to be a minimizer of $f$ is

$$
\partial f(x) \ni 0 .
$$

Unless $f$ is convex the above is not a sufficient condition. In the remainder, a point $x \in \mathbb{R}^{n}$ that satisfies (1) is called limiting-critical or critical. The set of critical points of $f$ is denoted by crit $f$.

\subsection{Proximal algorithm}

Let $f: \mathbb{R}^{n} \rightarrow \mathbb{R} \cup\{+\infty\}$ be a proper lower semicontinuous function. Given $x^{0} \in \mathbb{R}^{n}$ we consider the following discrete dynamical system

$$
x^{k+1} \in \operatorname{argmin}\left\{f(u)+\frac{1}{2 \lambda_{k}}\left|u-x^{k}\right|^{2}: u \in \mathbb{R}^{n}\right\},
$$

where $\left(\lambda_{k}\right)_{k \in \mathbb{N}}$ is a positive sequence.

Necessary and sufficient conditions for this algorithm to be well-defined can be found in RockafellarWets [24, Exercise 1.24., p. 20]. We simply assume here that

$$
\left(\mathcal{H}_{1}\right) \inf _{\mathbb{R}^{n}} f>-\infty
$$

which clearly implies that, for all $k \in \mathbb{N}$, the set appearing in (2) is nonempty and compact. Writing down the optimality condition [24, Theorem 10.1] and using the subdifferentiation formula for a sum of functions [24, Exercise 10.10]), it follows that there exists $g^{k+1} \in \partial f\left(x^{k+1}\right)$ such that

$$
x^{k+1}=x^{k}-\lambda_{k} g^{k+1} .
$$

Let us fix some positive parameters $\lambda_{-}$and $\lambda_{+}$with $0<\lambda_{-}<\lambda_{+}<+\infty$.

¿From now on we assume that $\lambda_{k} \in\left(\lambda_{-}, \lambda_{+}\right)$for all $k \in \mathbb{N}$.

Consider the following assumption:

$\left(\mathcal{H}_{2}\right)$ The restriction of $f$ to its domain is a continuous function (on $\operatorname{dom} f$ ).

The following result gathers a few elementary facts concerning the dynamical system (2).

Proposition 2 Let $\left(x^{k}\right)_{k \in \mathbb{N}}$ be a sequence which complies with (2) and denote by $\omega\left(x^{0}\right)$ the set of its limit points. Then

(i) The sequence $\left(f\left(x^{k}\right)\right)_{k \in \mathbb{N}}$ is decreasing,

(ii) $\sum\left|x^{k+1}-x^{k}\right|^{2}<+\infty$,

(iii) If $f$ satifies $\left(\mathcal{H}_{2}\right)$ then $\omega\left(x^{0}\right) \subset \operatorname{crit} f$.

If, in addition, the sequence $\left(x^{k}\right)_{k \in \mathbb{N}}$ is bounded then

(iv) $\omega\left(x^{0}\right)$ is a nonempty compact connected set, and

$$
d\left(x^{k}, \omega\left(x^{0}\right)\right) \rightarrow 0 \text { as } k \rightarrow+\infty,
$$

(v) If $f$ satifies $\left(\mathcal{H}_{2}\right)$ then $f$ is finite and constant on $\omega\left(x^{0}\right)$. 
Sketch of the proof Let us prove (i) and (ii). By definition, (2) implies that for all $k \geq 0$ we have

$$
f\left(x^{k+1}\right)+\frac{1}{2 \lambda_{k}}\left|x^{k+1}-x^{k}\right|^{2} \leq f\left(x^{k}\right) .
$$

This means that $f\left(x^{k}\right)$ is nonincreasing and by summing the inequalities (4) from 0 to $N \geq 0$ we also obtain that

$$
\sum_{k=0}^{N}\left|x^{k+1}-x^{k}\right|^{2} \leq 2 \lambda_{+}\left[f\left(x^{0}\right)-f\left(x^{N+1}\right)\right] \leq 2 \lambda_{+}\left[f\left(x^{0}\right)-\inf _{\mathbb{R}^{n}} f\right]<\infty .
$$

Let us deal with (iii) and (v). For any limit point $\bar{x}$ of $f$, we can use the lower semicontinuity of $f$ to obtain that $\lim _{k \rightarrow \infty} f\left(x^{k}\right) \geq f(\bar{x})$. If, in addition, $f$ satisfies $\left(\mathcal{H}_{2}\right)$ then the above inequality is actually an equality and (v) is proved. By using (ii), (3) and the fact that $\lambda_{k} \geq \lambda_{-}>0$ we can assume that there exists $k_{p} \rightarrow+\infty$ such that $\left\{\left(x^{k_{p}}, g^{k_{p}}\right)\right\} \rightarrow(\bar{x}, 0)$ with $f\left(x^{k_{p}}\right) \rightarrow f(\bar{x})$. Owing to the definition of the limiting subdifferential it follows that $(\bar{x}, 0)$ belongs to the graph of $\partial f$, so that (v) and (iii) are proved.

Item (iv) follows by using (ii) together with some classical properties of sequences in $\mathbb{R}^{n}$.

Remark 3 When $x^{k}$ is bounded, the convergence of the whole sequence $x^{k}$ may fail even for a finitevalued smooth function $f$, see Palis-De Melo [19] or Absil-Mahony-Andrews [1].

\section{Convergence analysis}

\section{$3.1 \quad$ Łojasiewicz inequality}

Let $f: \mathbb{R}^{n} \rightarrow \mathbb{R} \cup\{+\infty\}$ be a proper lower semicontinuous function that satisfies $\left(\mathcal{H}_{2}\right)$. The function $f$ is said to have the Łojasiewicz property if:

$\left(\mathcal{H}_{3}\right) \quad$ For any limiting-critical point $\hat{x}$, that is $\partial f(\hat{x}) \ni 0$, there exist $C, \epsilon>0$ and $\theta \in[0,1)$ such that

$$
|f(x)-f(\hat{x})|^{\theta} \leq C\left|x^{*}\right|, \forall x \in B(\hat{x}, \epsilon), \forall x^{*} \in \partial f(x) .
$$

Remark 4 When $\theta=0$ we adopt the convention $0^{0}=0$, and therefore if $|f(x)-f(\hat{x})|^{0}=0$ we have $f(x)=f(\hat{x})$.

Lemma 3 Assume that $f$ has the Lojasiewicz property.

(i) If $K$ is a connected subset of the set of critical points of $f$, that is $\partial f(x) \ni 0$ for all $x \in K$, then $f$ is constant on $K$.

(ii) If in addition $K$ is a compact set, then there exist $C, \epsilon>0$ and $\theta \in[0,1)$ such that

$$
\forall x \in \mathbb{R}^{n}, d(x, K) \leq \epsilon, \forall x^{*} \in \partial f(x), \quad|f(x)-f(\hat{x})|^{\theta} \leq C\left|x^{*}\right|
$$

Proof. Item (i) is a straightforward consequence of (ii), let us therefore deal with (ii). The compact set $K$ can be covered by a finite number of open balls $B\left(x_{i}, \epsilon_{i}\right)$, with $x_{i} \in K(i=1, \ldots, p)$ on which (5) holds with constants $C_{i}, \theta_{i}$. In other words, for each $i \in\{1, \ldots, p\}$ and for each $x \in B\left(x_{i}, \epsilon_{i}\right)$ we have

$$
\left|f(x)-f\left(x_{i}\right)\right|^{\theta_{i}} \leq C_{i}\left|x^{*}\right|
$$

for all $x^{*}$ in $\partial f(x)$. As a consequence, $f$ is locally constant (and continuous) on the connected set $K$, it is therefore constant. By choosing $\epsilon>0$ sufficiently small, we obtain that

$$
\left\{x \in \mathbb{R}^{n}: d(x, K) \leq \epsilon\right\} \subset \cup_{i=1}^{p} B\left(x_{i}, \epsilon_{i}\right),
$$

and the claimed result holds by letting $C=\max C_{i}$ and $\theta=\max \theta_{i}$. 
Remark 5 (a) Real-analytic functions have the Łojasiewicz property, see Łojasiewicz [17, 18].

(b) If $f$ is subanalytic and satisfies $\left(\mathcal{H}_{2}\right)$ (with $\operatorname{dom} f$ closed in $\mathbb{R}^{n}$ ), in particular if $f$ is continuous and subanalytic, it has the Łojasiewicz property, see [9, 10], and also Kurdyka-Parusinski [16] for similar results. Basic definitions and some examples of subanalytic functions can be found in [9]; for a comprehensive account on the topic one is referred to $[26,12]$ and references therein.

(c) Convex functions satisfying the following growth conditions

$$
\forall \hat{x} \in \operatorname{argmin} f, \exists C>0, r \geq 1, \epsilon>0, \forall x \in B(\hat{x}, \epsilon), f(x) \geq f(\hat{x})+C d(x, \operatorname{argmin} f)^{r}
$$

comply with (5) (with $\left.\theta=1-\frac{1}{r}\right)$, see [9].

(d) Infinite-dimensional versions of (5) have been developed in view of the asymptotic analysis of dissipative evolution equations. These can be found in Simon [25], and Haraux [14].

(e) Kurdyka has recently established a Łojasiewicz-like inequality for functions definable in an arbitrary o-minimal structure [15].

\subsection{Convergence results}

The proofs we develop here are adapted from Łojasiewicz's original idea [17].

Theorem 4 (Convergence result) Assume that $f$ satisfies $\left(\mathcal{H}_{1}\right),\left(\mathcal{H}_{2}\right),\left(\mathcal{H}_{3}\right)$ and let $\left(x^{k}\right)_{k \in \mathbb{N}}$ be a sequence generated by the proximal algorithm.

If the sequence $\left(x^{k}\right)_{k \in \mathbb{N}}$ is bounded, then

$$
\sum_{k=0}^{+\infty}\left|x^{k+1}-x^{k}\right|<+\infty
$$

in particular the whole sequence $\left(x^{k}\right)_{k \in \mathbb{N}}$ converges to some critical point of $f$.

Proof. Changing $f$ into $f-\inf _{k \geq 0} f\left(x^{k}\right)$ we can assume with no loss of generality that $f\left(x^{k}\right)$ converges to 0 . The case when $x^{k+1}=x^{k}$ for some $k \geq 1$ has no consequence on the asymptotic analysis, so that we may suppose that $\left|x^{k+1}-x^{k}\right|>0$ for all $k \geq 0$. In view of (4), we obtain also that $f\left(x^{k}\right)$ is positive and decreases (strictly) to 0 .

By using the convex inequality for the function $s>0 \rightarrow-s^{1-\theta}$ and (4) for all $k \geq 0$ we obtain that

$$
\begin{aligned}
f\left(x^{k}\right)^{1-\theta}-f\left(x^{k+1}\right)^{1-\theta} & \geq(1-\theta) f\left(x^{k}\right)^{-\theta}\left(f\left(x^{k}\right)-f\left(x^{k+1}\right)\right) \\
& \geq(1-\theta) f\left(x^{k}\right)^{-\theta} \frac{1}{2 \lambda_{k}}\left|x^{k+1}-x^{k}\right|^{2} .
\end{aligned}
$$

By Proposition 2 (iii) and (iv), and Lemma 3 (take $\left.K=\omega\left(x_{0}\right)\right)$ there exist an integer $N_{0}$, real numbers $C$ and $\theta \in(0,1)$ such that

$$
0<\left|f\left(x^{k}\right)\right|^{\theta} \leq C\left|g^{k}\right|=\frac{C}{\lambda_{k}}\left|x^{k}-x^{k-1}\right|
$$

for all $k \geq N_{0}$.

Combining the above result with (7) (recall $\lambda_{k}>\lambda_{-}>0$ ) yields the existence of a positive constant $M$ such that

$$
\frac{\left|x^{k+1}-x^{k}\right|^{2}}{\left|x^{k}-x^{k-1}\right|} \leq M\left(f\left(x^{k}\right)^{1-\theta}-f\left(x^{k+1}\right)^{1-\theta}\right)
$$

for all $k \geq N_{0}$.

Fix $r \in(0,1)$ and take $k \geq N_{0}$. If $\left|x^{k+1}-x^{k}\right| \geq r\left|x^{k}-x^{k-1}\right|$, (8) implies that

$$
\left|x^{k+1}-x^{k}\right| \leq \frac{M}{r}\left[f\left(x^{k}\right)^{1-\theta}-f\left(x^{k+1}\right)^{1-\theta}\right],
$$


and thus we have for all $k \geq N_{0}$

$$
\left|x^{k+1}-x^{k}\right| \leq r\left|x^{k}-x^{k-1}\right|+\frac{M}{r}\left[f\left(x^{k}\right)^{1-\theta}-f\left(x^{k+1}\right)^{1-\theta}\right] .
$$

If $N \geq N_{0}$ an easy induction yields

$$
\sum_{k=N_{0}}^{N}\left|x^{k+1}-x^{k}\right| \leq \frac{r}{1-r}\left|x^{N_{0}}-x^{N_{0}-1}\right|+\frac{M}{r(1-r)}\left[f\left(x^{N_{0}}\right)^{1-\theta}-f\left(x^{N+1}\right)^{1-\theta}\right],
$$

and the conclusion follows from the fact that $f$ is bounded from below.

Remark 6 Similar convergence results could be obtained for functions belonging to some o-minimal structure, see Kurdyka [15] and references therein.

If $\left(x^{k}\right)_{k \in \mathbb{N}}$ is a bounded sequence generated by (2), let us denote by $x^{\infty}$ its (unique) limit point. Applying Proposition (2) (iii) and (5) we obtain the existence of a neighborhood around $x^{\infty}$ such that (5) holds. The number $\theta$ appearing in (5) is then called a tojasiewicz exponent of $x^{\infty}$.

Theorem 5 (Rate of convergence) The assumptions are those of Theorem 4. Let $\left(x^{k}\right)_{k \in \mathbb{N}}$ be a bounded sequence generated by the proximal algorithm and let us denote by $\theta$ a Eojasiewicz exponent of $x^{\infty}$. The following estimations hold

(i) If $\theta=0$, the sequence $\left(x^{k}\right)_{k \in \mathbb{N}}$ converges in a finite number of steps,

(ii) If $\theta \in\left(0, \frac{1}{2}\right]$ then there exist $c>0$ and $Q \in[0,1)$ such that

$$
\left|x^{k}-x^{\infty}\right| \leq c Q^{k},
$$

(iii) If $\theta \in\left(\frac{1}{2}, 1\right)$ then there exists $c>0$ such that

$$
\left|x^{k}-x^{\infty}\right| \leq c k^{-\frac{1-\theta}{2 \theta-1}} .
$$

Proof The notations are those of the previous proof. For any $k \geq 0$, set $\Delta_{k}=\sum_{p=k}^{\infty}\left|x^{p+1}-x^{p}\right|$ which is finite by Theorem 4 . The triangle inequality yields $\Delta_{k} \geq\left|x^{k}-x^{\infty}\right|$, it is therefore sufficient to establish the estimations appearing in (ii) and (iii) for $\Delta_{k}$. With no loss of generality we may assume that $\Delta_{k}>0$ for all $k \geq 0$.

Using (9), and the fact that $f\left(x^{k}\right)$ decreases to zero we obtain for $k$ sufficiently large (recall that $r \in(0,1)$ can be taken arbitrarily)

$$
\begin{aligned}
\Delta_{k} & \leq \frac{1}{1-r}\left(\Delta_{k-1}-\Delta_{k}\right)+\frac{M}{r(1-r)} f\left(x^{k}\right)^{1-\theta} \\
& \leq \frac{1}{1-r}\left(\Delta_{k-1}-\Delta_{k}\right)+\frac{M}{r(1-r)}\left(C\left|g^{k}\right|\right)^{\frac{1-\theta}{\theta}} \\
& \leq \frac{1}{1-r}\left(\Delta_{k-1}-\Delta_{k}\right)+\left(\lambda_{-}\right)^{1-1 / \theta} \frac{M C^{\frac{1-\theta}{\theta}}}{r(1-r)}\left(\Delta_{k-1}-\Delta_{k}\right)^{\frac{1-\theta}{\theta}}
\end{aligned}
$$

where (10) and (11) follow respectively from (5) and (3).

Assume that $\theta$ belongs to $\left(\frac{1}{2}, 1\right)$, so that $\frac{1-\theta}{\theta}<1$. Since $\Delta_{k} \rightarrow 0$ as $k \rightarrow \infty$, we deduce from (11) that there exist an integer $N_{1} \geq N_{0}$ and a positive constant $C_{1}$ such that

$$
\Delta_{k}^{\frac{\theta}{1-\theta}} \leq C_{1}\left(\Delta_{k-1}-\Delta_{k}\right)
$$


for all $k \geq N_{1}$. Define $h:(0,+\infty) \rightarrow \mathbb{R}$ by $h(s)=s^{-\frac{\theta}{1-\theta}}$ and let $R \in(1,+\infty)$. Take $k \geq N_{1}$ and assume first that $h\left(\Delta_{k}\right) \leq R h\left(\Delta_{k-1}\right)$. By rewriting (12) as

$$
1 \leq \frac{C_{1}\left(\Delta_{k-1}-\Delta_{k}\right)}{\Delta_{k}^{\frac{\theta}{1-\theta}}},
$$

we obtain that

$$
\begin{aligned}
1 & \leq C_{1}\left(\Delta_{k-1}-\Delta_{k}\right) h\left(\Delta_{k}\right) \\
& \leq R C_{1}\left(\Delta_{k-1}-\Delta_{k}\right) h\left(\Delta_{k-1}\right) \\
& \leq R C_{1} \int_{\Delta_{k}}^{\Delta_{k-1}} h(s) d s \\
& \leq R C_{1} \frac{1-\theta}{1-2 \theta}\left[\Delta_{k-1}^{\frac{1-2 \theta}{1-\theta}}-\Delta_{k}^{\frac{1-2 \theta}{1-\theta}}\right] .
\end{aligned}
$$

Thus if we set $\mu=\frac{2 \theta-1}{(1-\theta) R C_{1}}>0$ and $\nu=\frac{1-2 \theta}{1-\theta}<0$ one obtains that

$$
0<\mu \leq \Delta_{k}^{\nu}-\Delta_{k-1}^{\nu}
$$

Assume now that $h\left(\Delta_{k}\right)>R h\left(\Delta_{k-1}\right)$ and set $q=\left(\frac{1}{R}\right)^{\frac{1-\theta}{\theta}} \in(0,1)$. It follows immediately that $\Delta_{k} \leq$ $q \Delta_{k-1}$ and furthermore - recalling that $\nu$ is negative - we have

$$
\begin{aligned}
\Delta_{k}^{\nu} & \geq q^{\nu} \Delta_{k-1}^{\nu} \\
\Delta_{k}^{\nu}-\Delta_{k-1}^{\nu} & \geq\left(q^{\nu}-1\right) \Delta_{k-1}^{\nu} .
\end{aligned}
$$

Since $q^{\nu}-1>0$ and $\Delta_{p} \rightarrow 0^{+}$as $p \rightarrow+\infty$, there exists $\bar{\mu}>0$ such that $\left(q^{\nu}-1\right) \Delta_{p-1}^{\nu}>\bar{\mu}$ for all $p \geq N_{1}$. Therefore we obtain that

$$
\Delta_{k}^{\nu}-\Delta_{k-1}^{\nu} \geq \bar{\mu}
$$

If we set $\hat{\mu}=\min \{\mu, \bar{\mu}\}>0$, one can combine (14) and (13) to obtain that

$$
\Delta_{k}^{\nu}-\Delta_{k-1}^{\nu} \geq \hat{\mu}>0
$$

for all $k \geq N_{1}$. By summing those inequalities from $N_{1}$ to some $N$ greater than $N_{1}$ we obtain that $\Delta_{N}^{\nu}-\Delta_{N_{1}}^{\nu} \geq \hat{\mu}\left(N-N_{1}\right)$ and consequently (iii) follows from

$$
\Delta_{N} \leq\left[\Delta_{N_{1}}^{\nu}+\hat{\mu}\left(N-N_{1}\right)\right]^{1 / \nu} \leq c N^{-\frac{1-\theta}{2 \theta-1}}(c \text { being a positive constant }) .
$$

When $\theta \in\left(0, \frac{1}{2}\right],(11)$ shows that $\Delta_{k}$ complies with the following inequality (for $k$ sufficiently large)

$$
\Delta_{k} \leq C_{2}\left(\Delta_{k-1}-\Delta_{k}\right),
$$

where $C_{2}$ is a positive constant. This implies that $\Delta_{k} \leq \frac{C_{2}}{1+C_{2}} \Delta_{k-1}$ and item (ii) follows easily with $Q=\frac{C_{2}}{1+C_{2}} \in(0,1)$.

Assume that $\theta=0$, set $I:=\left\{k \in \mathbb{N}: x_{k+1} \neq x_{k}\right\}$ and take $k$ in $I$. If $k$ is sufficiently large we have

$$
\frac{1}{\lambda_{k}^{2}}\left|x^{k+1}-x^{k}\right|^{2}=\left|g^{k+1}\right|^{2} \geq C_{3}>0,
$$


so that (4) implies that

$$
f\left(x^{k+1}\right) \leq f\left(x^{k}\right)-\frac{1}{2 \lambda_{k}}\left|x^{k+1}-x^{k}\right|^{2} \leq f\left(x^{k}\right)-C_{3} \frac{\lambda_{-}}{2} .
$$

Since $f\left(x_{k}\right)$ is known to converge to zero the above inequality clearly implies that $I$ is finite and (i) follows immediately.

\section{References}

[1] Absil, P.-A., Mahony, R. \& Andrews, B., Convergence of the Iterates of Descent Methods for Analytic Cost Functions, preprint 16p, 2004.

[2] Alvarez, F., Atтouch, H., An inertial proximal method for maximal monotone operators via discretization of a nonlinear oscillator with damping, Set-Valued Analysis, 9 (2001), 3-11.

[3] Attouch, H., Goudou, X., Redont, P., The heavy ball with friction method. The continuous dynamical system. Global exploration of the local minima of a real-valued function by asymptotic analysis of a dissipative dynamical system, Commun. Contemp. Math., 2 (2000), 1-34.

[4] Attouch, H., Soubeyran, A., Inertia and reactivity in decision making as cognitive variational inequalities, J. of Convex Analysis, (2005), to appear.

[5] Atrouch, H., Teboulle, M. Regularized Lotka-Volterra Dynamical system as continuous proximal-like method in optimization, J. Optim. theory Appl. 121 (2004), 541-580.

[6] Atrouch, H., Soubeyran, A., From procedural rationality to routines: a worthwhile to move approach of satisficing with not too much sacrificing, submitted paper, 2005.

[7] Benedetti, R. \& Risler, J.-J., Real Algebraic and Semialgebraic Sets, Hermann, Éditeurs des sciences et des Arts, (Paris, 1990).

[8] Bochnak, J., Coste, M. \& Roy, M.-F., Real Algebraic Geometry, (Springer, 1998).

[9] Bolte, J, Danillidis, A. \& Lewis, A., The Łojasiewicz inequality for nonsmooth subanalytic functions with applications to subgradient dynamical systems, submitted for publication.

Available electronically at: http://pareto.uab.es/〜adaniilidis/

[10] Bolte, J, Danillidis, A. \& Lewis, A., The Morse-Sard theorem for non-differentiable subanalytic functions, to appear in J. Math. Analysis \& Applications (2005).

[11] Clarke, F.H., Ledyaev, Yu., Stern, R.I. \& Wolenski, P.R., Nonsmooth analysis and control theory, Graduate texts in Mathematics 178, (Springer-Verlag, New-York, 1998).

[12] VAn den Dries, L. \& Miller, C., Geometric categories and o-minimal structures, Duke Math. J. 84 (1996), 497-540.

[13] Goudou,X., Munier, J. The heavy ball with friction method: the quasiconvex case, submitted, 2005.

[14] Haraux, A., A hyperbolic variant of Simon's convergence theorem. Evolution equations and their applications in physical and life sciences (Bad Herrenalb, 1998), Lecture Notes in Pure and Appl. Math. 215 (2001), 255-264 (Dekker, New York).

[15] Kurdyka, K., On gradients of functions definable in o-minimal structures, Ann. Inst. Fourier 48 (1998), 769-783. 
[16] Kurdyka, K. \& Parusinski, A., $\mathrm{w}_{f}$-stratification of subanalytic functions and the Eojasiewicz inequality, C. R. Acad. Paris 318 (1994), 129-133.

[17] Łojasiewicz, S., "Une propriété topologique des sous-ensembles analytiques réels.", in: Les Équations aux Dérivées Partielles, pp. 87-89, Éditions du Centre National de la Recherche Scientifique, Paris 1963.

[18] Łojasiewicz, S., Sur la géométrie semi- et sous-analytique, Ann. Inst. Fourier 43 (1993), 15751595.

[19] Palis, J. \& De Melo, W., Geometric theory of dynamical systems. An introduction, (Translated from the Portuguese by A. K. Manning), Springer-Verlag, New York-Berlin, 1982.

[20] Lemaire, B., The proximal algorithm. New methods in optimization and their industrial uses (Pau/Paris, 1987), 73-87, Internat. Schriftenreihe Numer. Math. 87, (Birkhäuser, Basel, 1989).

[21] Martinet, B. Régularisation d'inéquations variationnelles par approximations successives. (French) Rev. Française Informat. Recherche Opérationnelle 4 (1970), Ser. R-3, 154-158.

[22] Mordukhovich, B., Maximum principle in the problem of time optimal response with nonsmooth constraints, J. Appl. Math. Mech., 40 (1976), 960-969 ; [translated from Prikl. Mat. Meh. 40 (1976), 1014-1023]

[23] Rockafellar, R. T. Augmented Lagrangians and applications of the proximal point algorithm in convex programming, Math. Oper. Res. 1 (1976), no. 2, 97-116.

[24] Rockafellar, R.T. \& Wets, R., Variational Analysis, Grundlehren der Mathematischen, Wissenschaften, Vol. 317, (Springer, 1998).

[25] Simon, L., Asymptotics for a class of non-linear evolution equations, with applications to geometric problems, Ann. Math. 118 (1983), 525-571.

[26] Shiota, M, Geometry of subanalytic and semialgebraic sets, Progress in Mathematics 150, Birkhäuser, (Boston, 1997). 